\title{
Local plus sedoanalgesia or spinal analgesia in endovascular aortic aneurysm repair experiences in a single center
}

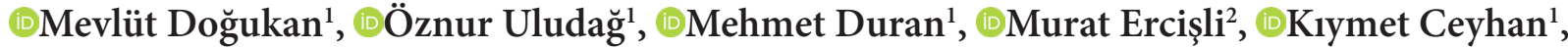 \\ (D)Cengiz Güven², (D)Ayşe Baysal ${ }^{3}$ \\ ${ }^{1}$ Adiyaman University Medical School, Department of Anesthesiology and Reanimation, Adiyaman, Turkey \\ ${ }^{2}$ Adiyaman University, Faculty of Medicine,Cardio-Vascular Surgery Department, Adıyaman, Turkey \\ ${ }^{3}$ Pendik Bölge Hospital, Clinic of Anesthesiology and Reanimation, Pendik, İstanbul, Turkey
}

Cite this article as: Doğukan M, Uludağ Ö, Duran M, et al. Local plus sedoanalgesia or spinal analgesia in endovascular aortic aneurysm repair experiences in a single center. Anatolian Curr Med J 2021; 3(2); 120-123.

\begin{abstract}
Aim: In this study, we aimed to discuss the local anesthesia plus sedoanalgesia (LA-SA) and spinal anesthesia methods applied during endovascular aortic repair (EVAR) by retrospectively comparing the patients' data.

Material and Method: This study was carried out by retrospectively evaluating the data of 36 patients, who underwent endovascular surgery for aortic aneurysm, between 1 January 2013 and 31 December 2018. Three cases who were applied general anesthesia were not included in the study.The patients included in the study were divided into two groups according to the anesthesia method as LA-SA group (Group 1, n=19) and spinal anesthesia group (Group 2, n=14). This study was planned as a retrospective observational comparative study. Demographic data, comorbidities, American Anesthesiologists Association (ASA) risk classification scores, mortality rates, duration of anesthesia and surgery, length of stay in the hospital and intensive care unit, and laboratory values were analyzed.

Results: In current study, the mean age of 33 patients who underwent EVAR procedure was $69.04 \pm 13$ (32-86). Local anesthesia plus sedo-analgesia was applied to $19(52.7 \%)$ patients and spinal anesthesia was applied to $14(38.8 \%)$ patients. No significant difference was found between demographic data, comorbidities and smoking rates in both groups. The length of stay in the hospital and the intensive care unit and the rates of death before discharge were similar in both groups $(\mathrm{p}=0.22),(\mathrm{p}=0.15)$, $(\mathrm{p}=0.73)$, Anesthesia and operation times were shorter in the local anesthesia plus sedoanalgesia group compared to the spinal anesthesia group ( $\mathrm{p}=0.00, \mathrm{p}=0.004$, respectively). Laboratory examinations of both groups were similar.

Conclusion: For arterial stents requiring percutaneous implantation, LA-SA provides a safe anesthesia method with stable hemodynamics, less invasive intervention and shorter operation times than neuraxial anesthesia.
\end{abstract}

Keywords: Endovascular aortic repair, local anesthesia sedoanalgesia, spinal anesthesia

\section{INTRODUCTION}

Abdominal aortic aneurysm (AAA) is very common among aortic pathologies. An enlargement of 1.5 times the normal segment or a total of more than $3 \mathrm{~cm}$ is considered an abdominal aortic aneurysm (1). The main risk factors for AAA can be stated as age, male gender, high blood pressure, smoking and family history (2). AAA is seen especially in the advanced age group. In the last thirty years, endovascular repair (EVAR) combined with neuraxial anesthesia or sedoanalgesia has been used instead of open surgery under general anesthesia, especially in patients with high complication rates (1). AAA's Endovascular Repair (EVAR) was introduced in 1990 to offer a lower-risk alternative to traditional open surgical repair (1). Although it was initially preferred in the patient group with high risk and systemic problems, many advantages such as providing lower mortality and morbidity rates, shortening the duration of hospital stay and reducing the use of blood products have enabled it to be used more and more (2-4). Sedoanalgesia is successfully used in EVAR $(4,5)$. Although general anesthesia was used more frequently in the first years, LA-SA is used more frequently today (6). Today, local anesthesia plus sedoanalgesia and spinal anesthesia have largely replaced general anesthesia. Both methods have advantages and disadvantages. In this study, it was aimed to compare the methods of LA-SA and spinal anesthesia applied to patients during endovascular aortic repair. 


\section{MATERIAL AND METHOD}

After the approval of the Ethics Committee of Adiyaman University Faculty of Medicine was obtained (Meeting: 4, Decision No: 2018/4-29), the patient files and anesthesia records of 36 patients who underwent EVAR procedure with the diagnosis of aortic aneurysmbetween 1 January 2013 and 31 December 2018 were evaluated retrospectively. The trial was conducted in accordance with the Helsinki Declaration principles. A total of 33 cases who underwent neuraxial anesthesia and LA-SA were included in the study for Endovascular Aortic Repair on the specified dates. Aortic dissections were not included in the study. Again, it was understood from the file scan that all patients were taken by the same surgical team. The patients were divided into two groups. The LA-SA Group (GroupLA-SA, $n=19$ ), and the Spinal Anesthesia Group (GroupSA, $n=14$ ). It was determined that the electrocardiography, peripheral oxygen saturation, and noninvasive blood pressure measurements of all cases were monitored routinely. In sedation administrations, in addition to local anesthesia by the surgeon in the form of skin infiltration, $0.05-0.1 \mathrm{mg} / \mathrm{kg}$ Midazolam (Zolamide, Vem, Turkey), and $0.5-1 \mu \mathrm{g} / \mathrm{kg}$ Fentanyl (Talinat ${ }^{\oplus}$, Vem, Turkey) were given intravenously (iv). The sedation scale of the patients was followed with the Ramsey-Sedation scale (RSS). No additional doses were administered to the patients with $\mathrm{RSS} \geq 4$. It was determined that the patients with RSS $<4$ were administered with $1 \mu \mathrm{g} / \mathrm{kg}$ (iv) Remifental (Ultiva ${ }^{\circ}$, GlaxoSmithKline, Belgium) in 30-60 seconds. In the patient who underwent LA-SA, $0.5-1 \mathrm{mg} /$ $\mathrm{kg}$ tramadol hydrochloride and $20 \mathrm{mg}$ Dexketoprofen were administered for postoperative analgesia. It was observed that $12.5 \mathrm{mg}$ Bupivacaine (Heavy Marcaine ${ }^{\oplus}$, Turkey), and $25 \mu \mathrm{g}$ Fentanyl were administered for spinal anesthesia. The sensory blocks of the patients were evaluated with the Pin-Prick test, and Motor Block Level Bromagescale.

The intraoperative heparin administration was delayed approximately 1 hour after the spinal needle was inserted in the Neuraxial Anesthesia Group. The patient files, demographic data of the patients, concomitant diseases, American Anesthesiologists Association risk classification scores, anesthesia and surgery duration, length of hospital and intensive care stay, mortality rates, preoperative and postoperative hemoglobin, hematocrit, platelet, mean platelet volume, urea, creatinine and albumin values were examined.

\section{Statistical Analysis}

The SPSS (Statistical Package for Social Sciences) and Windows 17.0 program were used for the statistical analyses. Numerical data were expressed as median, standard deviation, and categorical data as percentages. These patients were divided into two groups as local anesthesia plus sedoanalgesia and spinal anesthesia. Intragroup comparisons were made by repeated measures analysis. Chi-square test is used for the comparison of categorical data. Independent $t$ test was used to compare independent groups.p $<0.05$ was considered statistically significant.

\section{RESULTS}

In current study the mean age of 33 patients who underwent EVAR procedure was $69.04 \pm 13$ (3286). It was observed that local anesthesia plus sedoanalgesia was applied to $19(52.7 \%)$ patients and spinalanesthesia was applied to $14(38.8 \%)$ patients. No significant difference was found between the demographic data $(\mathrm{p}=0.77)$, comorbidities $(\mathrm{p}=0.61)$ and smoking rates $(\mathrm{p}=0.88)$ in both groups (Table 1). The length of stay in the hospital $(\mathrm{p}=0.22)$, the intensive care unit $(\mathrm{p}=0.15)$, and the pre-discharge rates $(p=0.73)$ were similar in both groups. The duration of anesthesia administration $(\mathrm{p}=0.001)$ and operation times $(\mathrm{p}=0.004)$ were statistically shorter in the localanesthesia plus sedoanalgesia group (Table 2). Compared to the preoperative values, postoperative hemoglobin, hematocrit, and albumin values were statistically lower $(\mathrm{p}<0.05)$, whereas MPV values were statistically higher for both groups $(\mathrm{p}<0.05)$ (Table 3$)$.

$\begin{aligned} & \text { Table 1. The comparison of the demographic data and additional } \\
& \text { diseases of the cases undergoing EVAR }\end{aligned}$
\begin{tabular}{|lccc|}
\hline Group & Group LA-SA n=19 & GroupSA n=14 & p \\
\hline $\begin{array}{l}\text { Gender } \\
\text { (Male/Female) }\end{array}$ & $14 / 5$ & $11 / 3$ & 0.75 \\
Age (year) & 72.83 & 65.28 & 0.77 \\
Hypertension & $15(78.9 \%)$ & $10(66.0 \%)$ & 0.61 \\
Diabetes & $5(26.3 \%)$ & $6(42.8 \%)$ & 0.33 \\
Pulmonary disease & $4(21.0 \%)$ & $2(40.0 \%)$ & 0.68 \\
Smoking & $14(73.6 \%)$ & $10(66.0 \%)$ & 0.88 \\
ASA II/III & $9 / 10$ & $7 / 7$ & 0.59 \\
\hline
\end{tabular}

LA-SA; localanesthesia plus sedoanalgesia, ASA; American Society of Aneshesiologists Chi-square was used for in-group comparisons

Table 2. The comparison of the cases undergoing EVAR in terms of hospital and intensive care unit stays, surgery durations and anesthesia durations

\begin{tabular}{|lccc|}
\hline Group & $\begin{array}{c}\text { Group LA-SA } \\
\mathbf{n = 1 9}\end{array}$ & $\begin{array}{c}\text { GroupSA } \\
\mathbf{n = 1 4}\end{array}$ & $\mathbf{p}$ \\
\hline Intensive care unit stay (day) & 1.4 & 1.2 & 0.22 \\
Hospital stay (day) & 6 & 5 & 0.15 \\
Anesthesia duration (minutes) & 135 & 151 & 0.01 \\
Surgery duration (minutes) & 122 & 138 & 0.04 \\
Mortality & 2 & 1 & 0.73 \\
\hline
\end{tabular}

$\mathrm{p}<0.05$ statistically significant LA-SA; localanesthesia plus sedoanalgesia, SA; Spinal anesthesia, Chi-square was used for in-group comparisons. 


\begin{tabular}{|c|c|c|c|c|}
\hline Group & Group LA-SA n=19 & $\mathbf{p}$ & Group SA n=14 & $\mathbf{p}$ \\
\hline Preop./Postop. Hgb & $11.9 \pm 1.6 / 10.9 \pm 1.4$ & 0.008 & $12.1 \pm 1.4 / 11.0 \pm 1.2$ & 0.007 \\
\hline Preop./Postop. Hct & $36.5 \pm 4.6 / 33.1 \pm 4.5$ & 0.001 & $36.8 \pm 4.2 / 34.4 \pm 4.3$ & 0.015 \\
\hline Preop./Postop. Plt & $259.3 \pm 50.0 / 238.4 \pm 65.9$ & 0.108 & $200.4 \pm 62.7 / 173.2 \pm 68.9$ & 0.204 \\
\hline Preop./Postop. Mpv & $6.9 \pm 1.3 / 7.4 \pm 1.7$ & 0.044 & $7.8 \pm 1.3 / 8.8 \pm 1.5$ & 0.015 \\
\hline Preop./Postop. Urea & $50.0 \pm 32.1 / 48.05 \pm 23.8$ & 0.747 & $46.2 \pm 23.3 / 50.7 \pm 32.2$ & 0.290 \\
\hline Preop./Postop. Cr & $1.0 \pm 0.7 / 1.1 \pm 0.4$ & 0.663 & $1.1 \pm 0.46 / 1.2 \pm 04$ & 0.054 \\
\hline Preop./Postop. Alb & $3.0 \pm 0.5 / 2.6 \pm 0.5$ & 0.009 & $3.2 \pm 0.5 / 2.9 \pm 0.5$ & 0.003 \\
\hline
\end{tabular}

\section{DISCUSSION}

Abdominal aortic aneurysms, especially aneurysm ruptures, are life-threatening events that have high mortality. The introduction of endovascular aneurysm repair, which was developed as an alternative to traditional open repairs, led to a decrease in mortality and morbidity in such patients. In early years, EVAR applications were mostly applied to a higher-risk patient group. Since endovascular repair of abdominal aortic aneurysms is carried out with a less invasive technique compared to conventional open surgical repair, a lower-risk treatment alternative has been introduced for many patients with aortic aneurysms and aortic dissection in recent years. Local anesthesia, regional anesthesia and general anesthesia techniques are used for this procedure. Since early procedures usually require long surgical durations, general anesthesia is applied to improve patient compliance.

The EVAR procedure carried out in our hospital was performed under LA-SA at a rate of $52.7 \%$, and under spinal anesthesia at a rate of $38.8 \%$. The demographic data of the patients in bothgroups, hospital and intensive care stays, mortality rates before discharge were found to be similar. Anesthesia and operation durations were statistically longer in the spinal anesthesia group than in LA-SA group. Hypotension developed in four patients (28.5\%) who underwent spinal anesthesia, and was resolved with the addition of $10 \mathrm{mg}$ iv ephedrine. No other complications or epidural hematoma occurred because of spinal anesthesia in the spinal group.

Unlike our study, general anesthesia was applied mostly in the first studies. In a wide scale article in 2006, the patients underwent general anesthesia (69\%), regional anesthesia (25\%), and local anesthesia (6\%). In the same study, it was shown that patients benefited more when local anesthesia technique was used for EVAR (7). In a literature review released in 2019, no differences were detected in mortality between local anesthesia for EVAR and general anesthesia group (8). In another article released in 2019, 30-day mortality of the patients who underwent general anesthesia was compared with local anesthesia group,and mortality was found to be less in the local anesthesia group (9). In another comprehensive article released in 2011, the anesthesia types were applied as general anesthesia (81\%), spinal anesthesia (7\%), epidural anesthesia (5.5\%), and local anesthesia (6.5\%). General anesthesia was used more in this study and general anesthesia was found to be associated with hospital stay and pulmonary morbidity compared to spinal and local anesthesia (10). Since the selection of anesthesia technique might be tolerated with neuraxial anesthesia, local anesthesia and sedation for arterial stents, which require percutaneous placement with limited incision, the general anesthesia application has gradually decreased. With increased experience of doctors, and with the development of more modern devices, the use of regional anesthetics as well as local anesthetics supported by mild sedation has increased (11-13).

In a study conducted in our country, a total of $77.1 \%$ of the patients underwent LA-SA, 22.8\% general anesthesia was applied, and it was reported that local anesthesia plus sedation provided safe and comfortable environment for endovascular applications (14). In another study, the intensive care and hospitalization duration of patients in the local anesthesia group were shorter than regional and general anesthesia groups (15). The incidence of respiratory complications is lower in local anesthesia compared to local anesthesia and general anesthesia in patients who undergo EVAR.

The use of procedures regarding neuraxial anesthesia in these patients must be conducted after careful riskbenefit analysis, and after strict and guideline-compliant discontinuation of anticoagulant therapy (16). When using neuraxial anesthesia, intraoperative heparin administration is postponed approximately 1 hour after the epidural catheter insertion because of epidural hematoma risk $(17,18)$. Although it is a rare complication of neuraxial blockade with epidural hematoma, neuraxial anesthesia is not applied in some centers because of the intraoperative heparin administration. In patients who undergo regional anesthesia, the request to wait 1 hour after the intraoperative heparin administration might affect the operation and anesthesia durations. In our study, the anesthesia and operation durations were statistically shorter in the LA-SA group than the regional anesthesia group. 
The selection of anesthesia technique might vary depending on the planned surgical interventions, comorbid conditions of the patient, preoperative and on the intraoperative anticoagulation use. LA-SAfor arterial stents, which require percutaneous insertion with limited incision, has been used as a safe anesthesia method in recent years, and is well-tolerated by the majority of patients. The retrospective natureof our study and the fact that very few cases were taken in our hospital (an average of 5-6 cases a year) were the limitations of this study.

\section{CONCLUSIONS}

For arterial stents requiring percutaneous placement with minimally invasive methods, LA-SA provides a safe anesthesia as a less invasive method that provides shorter operation times than stable hemodynamic and neuraxial anesthesia.

\section{ETHICAL DECLARATIONS}

Ethics Committee Approval: After the approval of the Ethics Committee of Adiyaman University Faculty of Medicine was obtained (Meeting: 4, Decision No: 2018/4-29).

Informed Consent: Because the study was designed retrospectively, no written informed consentform was obtained from patients.

Referee Evaluation Process: Externally peer-reviewed.

Conflict of Interest Statement: The authors have no conflicts of interest to declare.

Financial Disclosure: The authors declared that this study has received no financial support.

Author Contributions: All of the authors declare that they have all participated in the design, execution, and analysis of the paper, and that they have approved the final version.

\section{REFERENCES}

1. Parodi JC, Palmaz JC, Barone HD. Transfemoral intraluminal graft implantation for abdominal aortic aneurysms. Ann Vasc Surg 1991; 5: 491-9.

2. Jordan WD, Alcocer F, Wirthlin DJ, Westfall AO, Whitley D. Abdominal aortic aneurysms in "high-risk" surgical patients: comparison of open and endovascular repair. Ann Surg 2003; 237: 623-9.

3. Numan F, Gülşen F, Arbatlı H, Cantaşdemir M, Solak, S.New horizons in theendovascular treatment of aortic aneurysms. Turkish J ThoracCardiovasc Surg 2011; 19: 27-32.

4. Gürbüz A, Özsöyler İ, Yllık L, Güneş T, Aksun M, Uluç E. Combined surgical and endovascular repair of thoracic and thoraco abdominal aortic aneurysms. Turkish J Thorac Cardiovasc Surg 2008; 16: 146-9.

5. Gumus F, Polat A, Farsak B, Alagöl A. Anesthesia approach in endovascular aortic reconstruction. Koşuyolu Kalp Derg 2013; 16: $25-31$
6. Elisha S, Nagelhout J, Heiner J, Gabot M. Anesthesia case management for endovascular aortic aneurysm repair. AANA J. 2014; 82: 145-52.

7. Ruppert V, Leurs LJ, Steckmeier B, Buth J, Umscheid T. Influence of anesthesia type on outcome after endovascular aortic aneurysm repair: an analysis based on EUROSTAR data. J Vasc Surg. 2006; 44: 16-21.

8. Harky A, Grafton-Clarke C, Chan J. Is local anaesthesia superior to general anaesthesia in endovascular repair of abdominal aortic aneurysm? Interact Cardiovasc Thorac Surg 2019; 29: 599-603.

9. Armstrong RA, Squire YG, Rogers CA, Hinchliffe RJ, Mouton R. Type of Anesthesia for Endovascular Abdominal Aortic Aneurysm Repair. J Cardiothorac Vasc Anesth 2019; 33: 462-71.

10. Edwards MS, Andrews JS, Edwards AF, et al. Results of endovascular aortic aneurysm repair with general, regional, and local/monitored anesthesia care in the American College of Surgeons National Surgical Quality Improvement Program database. J Vasc Surg 2011; 54: 1273-82.

11. Henretta JP, Hodgson KJ, Mattos MA, et al. Feasibility of endovascular repair of abdominal aortic aneurysms with local anesthesia with intravenous sedation. J Vasc Surg 1999; 29: 793-8.

12. Mathes DD, Kern JA. Continuous spinal anesthetic technique for endovascular aortic stent graft surgery. J Clin Anesth 2000; 12: 487-90.

13. Lachat M, Pfammatter T, Turina M. Trans femoral end ografting of thoracic aortic aneurysm under local anesthesia: a simple, safe and fast track procedure. Vasa 1999; 28: 204-6.

14. Türktan M, Göçen U. Anesthesia approaches in endovascular aortic repair: single center experience. Cukurova Med J 2019; 44 1256-61.

15. Bettex DA, Lachat M, Pfammatter T, Schmidlin D, Turina MI, Schmid ER. To compare general, epidural and local anaesthesia for endovascular aneurysm repair (EVAR). Eur J Vasc Endovasc Surg 2001; 21: 179-84.

16. Rössel $\mathrm{T}$, Paul $\mathrm{R}$, Richter $\mathrm{T}$, et al. Management beiendovaskulärenGefäßeingriffen (Management of anesthesia in endovascular interventions). Anaesthesist. 2016; 65: 891-910.

17. Llau JV, De Andrés J, Gomar C, Gómez-Luque A, Hidalgo F, Torres LM. Anticlotting drugs and regional anaesthetic and analgesic techniques: comparative update of the safety recommendations. Eur J Anaesthesiol 2007; 24: 387-98.

18. Waurick K, Riess H, Van Aken H, Kessler P, Gogarten W, Volk T. S1- LeitlinieRückenmarksnaheregionalanästhesien und thrombembolieprophylaxe/antithrombotischemedikation. AnästhIntensivmed 2014; 55: 464-4. 